\title{
Sheath-core bicomponent fiber characterization by FT-IR and other analytical methodologies
}

\author{
Marcia Murakoshi Takematsu', Milton Faria Diniz², Elizabeth da Costa Mattos ${ }^{1,2}$ \\ and Rita de Cássia Lazzarini Dutra ${ }^{1 *}$
}

\author{
1'Instituto Tecnológico de Aeronáutica - ITA, São José dos Campos, SP, Brasil \\ 2Instituto de Aeronáutica e Espaço - IAE, Divisão de Química - AQI, São José dos Campos, SP, Brasil \\ *ritacld@ita.br
}

\begin{abstract}
The bicomponent fibers are a special class of fibers that consolidate two polymers in only one fiber in order to explore individual properties of each polymer and can be designed in a spatial configuration that allows the enhancement in application of this material. Thereby, an appropriate characterization of bicomponent fibers is very valuable to process monitoring, quality control and forensic investigation. The sheath-core bicomponent fiber composed by polyethylene (PE), polypropylene (PP) and titanium dioxide $\left(\mathrm{TiO}_{2}\right.$ ) was analyzed by Fourier transform infrared (FT-IR) spectroscopy and other analytical methodologies. Results obtained by FT-IR using modern accessories showed efficiency to characterize the polymers of sheath (PE) and core (PP), moreover these polymers were confirmed by DSC (Differential Scanning Calorimetry). The morphology and elemental composition were also studied by scanning electron microscopy with energy dispersive X-ray spectroscopy (SEM/EDS). The thermogravimetric analysis (TGA) and colorimetric analysis allowed characterize and quantify the concentration of $\mathrm{TiO}_{2}$.
\end{abstract}

Keywords: bicomponent fiber, characterization, FT-IR, polyethylene, polypropylene.

\section{Introduction}

The bicomponent fiber is produced to explore properties nonexistent in the fiber constituted by a unique polymer and is developed in order to achieve better results in these criteria: luster, resistance, dyeing, shrinkage, and stability of the fiber. The spatial configuration of two components of fiber consists usually of two types: side by side and sheath-core. In the first configuration, two components are divided lengthwise in two or more different regions and, the second type, one component is in the core of fiber and another one around the core and is called sheath ${ }^{[1]}$. These fibers are also known as "composite", "conjugate", and "hetero" fibers and, nowadays, the majority of bicomponent fibers commercially produced is sheath-core which assumes different spatial configurations depending on core position ${ }^{[2]}$.

Dasdemir et al. ${ }^{[2]}$ also describes that the major application of these fibers is in nonwoven area and one of purpose of use includes the increase of flexibility and strength, improvement of melting process, cost reduction and enhancement in surface properties of material.

Andrzejewski, et al. ${ }^{[3]}$ studied a new concept of bicomponent fibers as a base material for self-reinforced composite. The objective of this study was to explore the different thermal properties performed by bicomponent fibers when they are processed by different techniques, such as extrusion and injection molding.

The fibers are the main components of nonwoven and have performed an important role in mechanical properties $^{[4]}$. There are also additives able to modify the fibers to communicate and improve desirable properties or reduce and remove some unwelcome attributes to final product. One example of modification is the use of delustrant component which reduces the brightness as long as intensify the opacity of fiber. With this purpose, $\mathrm{TiO}_{2}$ can be incorporated as a concentrated suspension in the solid polymer before extrusion reducing significantly the transparence of fiber ${ }^{[5]}$. This present study was conducted to characterize the sheath-core bicomponent fibers constituted by thermoplastic polymers (PE/PP) and $\mathrm{TiO}_{2}$.

According to Demirci et al. ${ }^{[4]}$, the sheath-core bicomponent fibers have the sheath constitution a material with lower melting point than core material. In bicomponent fibers, $\mathrm{PE}$ is frequently used in the sheath whereas PP, polyamide 6 (PA6) and polyester, such as polyethylene terephthalate (PET) are the polymers most often used as core material. The polymers used in the core have usually the highest values of elasticity module than the sheath material and the transversal area of core is larger than the sheath.

One important polymer of great industrial importance is PE that is very used in the sheath because this polymer has played a good interfacial stability in bicomponent melt spun process due its thermal expansion and behavior very characteristic of $\mathrm{PE}^{[2]}$. Since 1950, the HDPE (high density polyethylene) is considered for perform superior mechanical properties in extrusion of melt fibers however the application of PP fibers in the nonwoven sector, particularly in medical and hygiene areas, had risen more recently ${ }^{[5]}$.

The most important polymer used in the world is the PP. However, PP presents hydrophobicity, poor biocompatibility 
and the low reactivity in the surface restricts its application. Many physical and chemical surface treatments were developed in order to turn the surface more reactive, such as: blending, surface coating, plasma treatment, gamma ray, UV (ultraviolet), ozonation treatment and chemical grafting ${ }^{[6]}$.

The different characteristics of materials used in bicomponent fibers composition and the importance of appropriate manufacturing to attend the required properties to final product, address to an ideal characterization of the components to verify the real composition and, significantly, the spatial configuration of fibers.

Other application of bicomponent fibers consists in the enhancement of the structure of disposable hygienic absorbent products, such as disposable diapers, especially in a layer that has property of liquid permeation. As the absorbent layer is typically made from an absorbent material that includes natural fibers such as cellulose, fluff pulp fibers, cotton fibers, and rayon fibers, and a super absorbent polymer (SAP), after liquid is absorbed into the absorbent layer, the absorbent material of the absorbent layer tends to expand and become heavy causing an uncomfortable feeling in user. In order to perform a supportive structure to fix the absorbent material in place, the synthetic fibers are incorporated into the absorbent layer ${ }^{[7]}$.

Several analytical techniques are applied to study these fibers. Meleiro and García-Ruiz ${ }^{[8]}$ conducted spectroscopic techniques in order to identify and compare different textile fibers with aim in forensic field. Microspectrophotometry in the ultraviolet-visible range and Raman spectroscopy were the main techniques studied in this scientific paper. They mentioned that UV-Vis microspectrophotometry is the first recommended technique, which is principally used to study the color of the fibers. Moreover, FT-IR spectroscopy is the recommended tool to determine the fiber nature that can be combined with Raman as a complementary technique to get a comprehensive analysis of textile fibers. Others analytical methodologies are emerging to the analysis of fibers traces, such as infrared (IR) chemical imaging spectroscopy and $\mathrm{X}$-ray fluorescence spectroscopy ${ }^{[8]}$.

Based on the combination of techniques, this present study is focused on characterize a bicomponent fiber by FT-IR spectroscopy associated with others different methodologies with purpose to contribute with new methodologies to forensic field, polymer and fiber characterization.

The FT-IR spectroscopy consists in one of many analytical tools that have been performed to characterize blends, especially when two polymers are in separate and distinct phases it is possible to assume that there are two different materials that not interact in IR spectrum, except in a possible interaction between the two phases. In the last case, the IR spectrum shows the bands derived of miscibility of polymers; on the contrary, it is feasible to determine the two polymers analyzing the blend by this technique ${ }^{[9]}$.

Prati et al. ${ }^{[10]}$ studied IR spectra obtained by FT-IR microscopy which allies the images to analytical features of FT-IR spectrophotometer. FT-IR microscopy can be used in reflection and transmission modes. The ATR (attenuated total reflectance) objective is generally considered one of the more reliable methods to analyze cross sectional areas, due to the fact of register similar spectra collected by transmission mode. There are two precautions to be taken when this FT-IR technique is used: the contact between the crystal and sample should be suitable and, as the sample can slide on the crystal, it is necessary to verify if the area to be analyzed do not move during the spectrum register. Flynn et al. ${ }^{[1]}$ concluded that IR chemical imaging with sample preparation using DAC (diamond Anvil cell) accessory and ATR microscopy provides the spatial configuration of bicomponent fibers and chemical information of each polymer that composes the fibers as well if it is possible to detect the spectral differences between the two presented components, once depending on the size of fiber diameter, the ATR microscopy does not allow spectral resolution to differ the polymers. For this reason, there are restrictions regarding to the ATR microscopy mode.

In this present study, three non-destructive FT-IR techniques were performed given that two by transmission mode (with and without microscope), PAS (photoacoustic spectroscopy) and reflection mode which consists of DRIFT (diffuse reflectance infrared Fourier transform) and UATR (universal attenuated total reflectance) in order to investigate the surface and core of bicomponent fiber. Basically, the study was conducted employing: SEM images to characterize the sheath-core configuration and width of each layer; thermal analysis to determine mass loss during thermal degradation; FT-IR and ultraviolet and visible (UV-VIS) spectroscopy to characterize known components. Related to inorganic content, $\mathrm{TiO}_{2}$, analysis by FT-IR transmission mode of fiber calcination residue were carried out with purpose of identify the type of $\mathrm{TiO}_{2}$ crystal added to polymer of the bicomponent fiber studied in this paper. Additionally to this, the bicomponent fibers were also studied by EDS to understand in which part of sheath-core bicomponent fiber the titanium was incremented and other pyrolysis reaction of inorganic residue with potassium pyrosulfate with after color reaction with hydrogen peroxide was also performed to confirm the presence of titanium and to quantify this residue.

\section{Materials and Methods}

\subsection{Sample}

The bicomponent fiber 3 denier PE/PP supplied by Far Eastern New Century Corporation (Taiwan) was used. The composition of this material is known: PE, PP, blue dye and $\mathrm{TiO}_{2}$. As the dye has minimum concentration in the fiber, this additive was not part of the scope of this study.

\subsection{Equipment/experimental conditions/methodologies}

\subsubsection{SEM / EDS}

The samples were analyzed using FEI Quanta600 FEG. The images were acquired in SE (secondary electron) mode and different magnifications were applied (500x and 9500x). The EDS analysis with an acceleration tension of $15 \mathrm{kV}$ was used in backscattering mode. The samples were fractured after freezing in liquid nitrogen and the sputtering with platinum was prepared before mounted on SEM stub. The intention of this experiment was to analyze qualitatively the sample to study the elemental composition in each part of fiber and define the cross-sectional area to understand the type of bicomponent fiber. Moreover, the SEM technique 
allows to measure each part of bicomponent fiber and this resource was also taken in one fiber to understand mainly the width of surface layer, once the sample was studied by FT-IR surface modes: UATR and PAS.

\subsubsection{DSC}

The fiber sample was analyzed by equipment of PerkinElmer DSC 6000 and about $1.5 \mathrm{mg}$ of sample was introduced into aluminum pan and then tightly sealed. One empty aluminum pan was used as a reference. With continuous nitrogen $\left(\mathrm{N}_{2}\right)$ flow, the pans were heated until $200{ }^{\circ} \mathrm{C}$ at $10^{\circ} \mathrm{C} / \mathrm{min}$ and then cooling step was conducted at $20^{\circ} \mathrm{C} / \mathrm{min}$ until $-40^{\circ} \mathrm{C}$, to arrange the crystals. After cooling, the second curve of melting was evaluated by new heating until $200{ }^{\circ} \mathrm{C}$ at $10^{\circ} \mathrm{C} / \mathrm{min}$. The apparatus calibration was carried out with indium and zinc as standards. As the first heating step presents the shape memory, the polymer melting temperatures $\left(\mathrm{T}_{\mathrm{m}}\right)$ were taken from the second heating curve.

\subsubsection{TGA}

Pyris 1 TGA model (PerkinElmer) was used to determine the content of inorganic residue in the sample. About $3.0 \mathrm{mg}$ of sample was weighed in the platinum crucible and heated until $800^{\circ} \mathrm{C}$ with a continuous $\mathrm{N}_{2}$ flow at $20^{\circ} \mathrm{C} / \mathrm{min}$; then the atmosphere was shifted to synthetic air until $1000^{\circ} \mathrm{C}$ at the same rate and the final temperature was hold for 2 minutes. The apparatus calibration was carried out with iron and nickel as standards.

\subsubsection{Determination of $\mathrm{TiO}_{2}$ through ultraviolet-visible (UV-VIS) spectroscopy}

8453 model UV-VIS spectrophotometer (Agilent) was used to determine the absorbance at $410 \mathrm{~nm}$ using $1 \mathrm{~cm}$ quartz cuvette. The sample was carbonized in Bunsen burner until form a black residue and it was taken to a muffle furnace at $800{ }^{\circ} \mathrm{C}$ for 3 hours. The white residue formed after $800{ }^{\circ} \mathrm{C}$ was pyrolyzed with potassium pyrosulfate in Bunsen burner until complete the reaction. The solid formed was dissolved with sulfuric acid $50 \%$ in water and the reaction with $30 \%$ hydrogen peroxide $\left(\mathrm{H}_{2} \mathrm{O}_{2}\right)$ was performed to achieve the yellow color to identify and quantify the titanium present in $\mathrm{TiO}_{2}$.

\subsubsection{FT-IR spectroscopy}

The transmission, reflection and photoacoustic modes were analyzed by Spectrum One. PAS mode (300 model supplied by MTEC, Inc.) and FT-IR microscope (FT-IR MIC) in transmission mode were conducted in Spectrum 2000 and AutoIMAGE PerkinElmer microscope, the both equipment were supplied by PerkinElmer.

PAS and reflection modes consist in FT-IR surface techniques whereas in transmission mode the IR beam pass through the sample and the spectrum represents all material that compose the sample. The reflection accessories used were: UATR and DRIFT. UATR consists of a composite $\mathrm{Zn}$-Se diamond crystal that allows collect the spectra directly from the surface sample without any special preparation. The UATR spectra were collected over the range $4000-550 \mathrm{~cm}^{-1}$ (MIR- middle infrared region), gain 1 and 20 scans at resolution of $4 \mathrm{~cm}^{-1}$. The background scan was obtained before the initiate the sample analysis and it was acquired without any material in contact with the crystal maintained dry and clean.
On the other hand, the reference material used to perform the background scan of DRIFT and PAS accessories were, respectively, calibration mirror and carbon black (supplied by MTEC Photoacoustic, Inc.). DRIFT spectra were collected over the range $4000-400 \mathrm{~cm}^{-1}$, gain $1,20 \%$ energy, 20 scans at resolution of $4 \mathrm{~cm}^{-1}$. PAS spectra were collected over the range $4000-600 \mathrm{~cm}^{-1}$, gain 1,32 scans at resolution of $4 \mathrm{~cm}^{-1}$, using helium gas as purge gas, mirror velocities to obtain the spectra: 0.05 and $1.00 \mathrm{~cm} / \mathrm{s}$. Transmission spectra were collected over the range $4000-400 \mathrm{~cm}^{-1}$, gain 1,20 scans at resolution of $4 \mathrm{~cm}^{-1}$.

The conditions of FT-IR MIC in transmission mode were range $4000-700 \mathrm{~cm}^{-1}$ and 20 scans at resolution of $4 \mathrm{~cm}^{-1}$.

The samples were prepared according to required preparation of each technique as following described:

- Transmission mode: this technique was used to identify the inorganic residue of sample. The fiber, as received, was prepared to be analyzed by transmission mode in this proportion 1:400 $\mathrm{mg} \mathrm{KBr}$ pellet. The calcination of bicomponent fiber in muffle furnace was conducted before the preparation of $\mathrm{KBr}$ pellet $(0.6: 400 \mathrm{mg} \mathrm{KBr}$ ). Moreover, the casting film was prepared dissolving about $20 \mathrm{mg}$ of sample previously in $20 \mathrm{~mL}$ toluene (lightly heated) and then the thin film was formed by evaporation of solvent in a watch glass.

- UATR mode: the area of crystal that had contact with the sample was approximately $2 \mathrm{~mm}$ of diameter. The sample area was visually selected and the pressure about $120 \mathrm{~N}$ was applied on the sample to ensure a good contact between the sample and incident IR beam, preventing loss of IR incident radiation.

- FT-IR MIC transmission mode: for transmission analysis using microscope, some fibers were disposed very straight in a support with a window to IR beam pass through the sample and acquire the IR spectrum. Background was collected adjacent to the sample through the air.

- DRIFT mode: the sample was tested directly in DRIFT mode using the macro sample support.

- PAS mode: the sample was also tested directly in this mode.

\section{Results and Discussions}

\subsection{Morphology and elemental identification of fibers by SEM/EDS}

The fibers were analyzed related to morphology and elemental identification as shown in Figure 1. The EDS detector coupled to SEM was used with objective to identify the chemical elements present in the layers of sample. A high accelerating voltage (generally higher than $10 \mathrm{kV}$ ) is required to excite the electrons of interest elements. This detector is largely used in material which composition has many elements ${ }^{[1]]}$. Previous studies including SEM analysis of bicomponent fibers focused only in physical structure of the material ${ }^{[1-3]}$ 
As from images obtained by aggregated fibers (Figure 1a) and the cross-sectional fiber it was possible to classify as sheath-core because two layers are evidenced by Figure 1b, in which there is an inner layer with approximately $16.78 \mu \mathrm{m}$ of diameter (core) with another one surrounded all inner layer with approximately $3.72 \mu \mathrm{m}$ of width (sheath) in a cylindrical format. In order to investigate the chemical composition in the core and sheath, the EDS spectra were collected in backscattering mode and then the elemental analysis was performed in two points in which the beam is irradiated on a chosen area within the field of view. In the region 1 (core), it was observed only presence of carbon (C) as shown image Figure 1c; however, in region 2 (sheath) carbon $(\mathrm{C})$, oxygen $(\mathrm{O})$ and titanium $(\mathrm{Ti})$ were identified according to Figure 1d. This technique SEM/EDS was also used in a recent paper to characterize iron as $\mathrm{Fe}_{3} \mathrm{O}_{4}$ nanoparticles present in bicomponent nanofibers composite ${ }^{[12]}$.

By EDS analysis it is possible to assure the presence of $\mathrm{Ti}$ and $\mathrm{O}$ in the sheath of bicomponent fibers indicating an increment of the additive $\mathrm{TiO}_{2}$ in the outside of fiber to give more opacity to material ${ }^{[5]}$. Nevertheless, more two studies were conducted to characterize and quantify this oxide in these fibers by colorimetric and thermal gravimetric methodologies. Otherwise, by FT-IR it was possible to characterize this inorganic additive by spectrum of calcinated residue. Once the EDS technique is dedicated to elemental analysis, the polymers that compose the bicomponent fiber can not be distinguished by this technique. For this reason, this study has proposed DSC analysis to find out the melting point of polymers and additional methodologies developed by FT-IR were performed with the purpose to enrich the characterization of polymers that compose each layer of bicomponent fiber.

\subsection{DSC analysis}

The polymers $T_{m}$ was taken by second heating curve and this curve can be observed in Figure 2. The thermogram indicates two melting peaks in the second curve at $128^{\circ} \mathrm{C}$ and $162{ }^{\circ} \mathrm{C}$ suggesting that the composition of the samples is based on PE and PP, once the $\mathrm{T}_{\mathrm{m}}$ of each polymer is according to the literature ${ }^{[13]}$. As from these results, it is possible to suggest that the composition of sample is $\mathrm{PE}$ and PP, as mentioned by supplier. However, more studies are necessary to confirm this characterization and further understand what polymer is related to sheath and core. In order to achieve these objectives, these studies were performed by FT-IR.

\subsection{TGA analysis}

This thermal analysis allowed investigating the content of material composition, such as: volatiles components: $0.135 \%$ until $300{ }^{\circ} \mathrm{C}$; polymeric degradation:
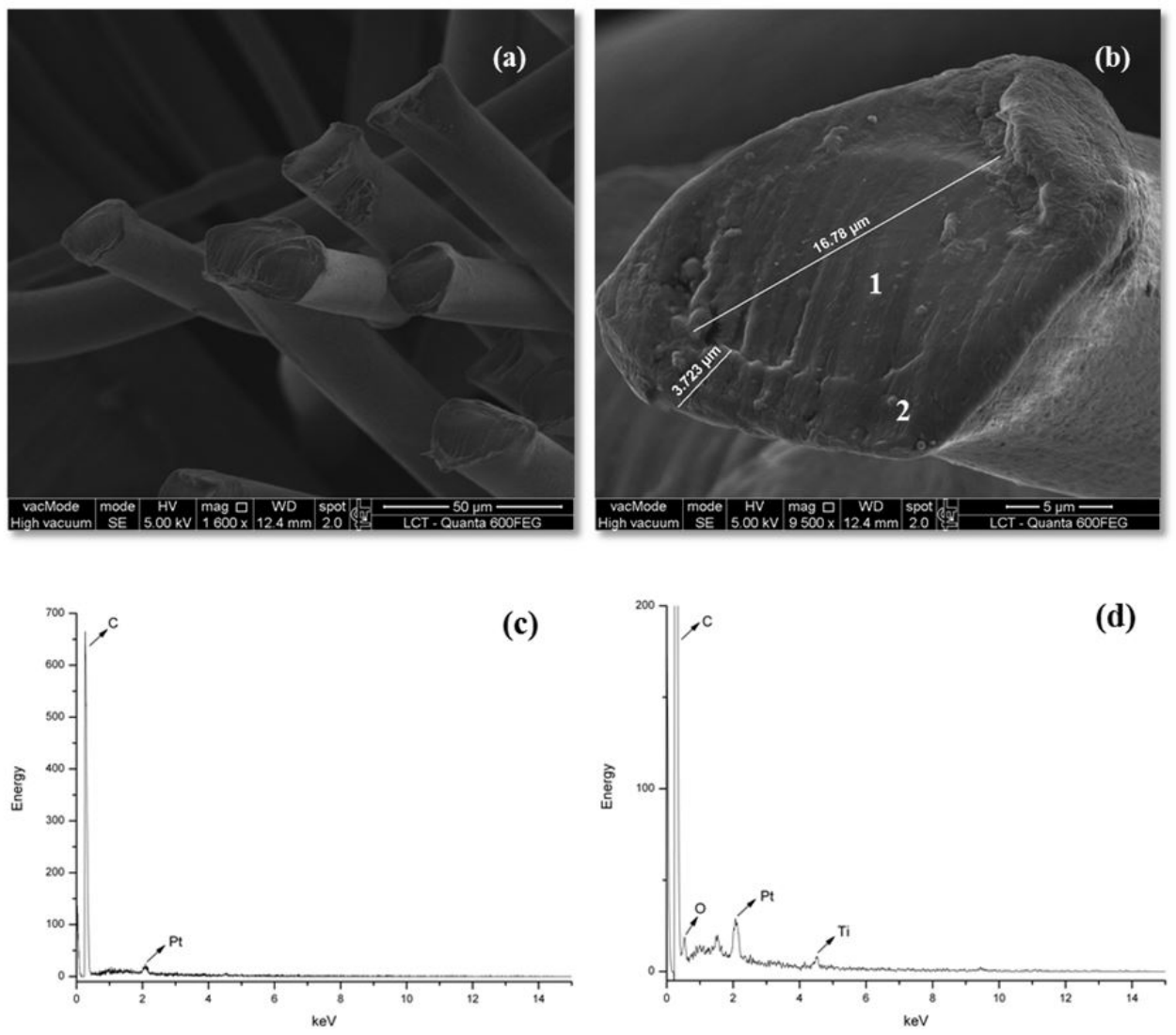

Figure 1. SEM images and EDS spectra of 3 denier PE/PP bicomponent fiber: (a) SEM micrograph of fibers; (b) SEM micrograph of individual fiber cross section with width of core and sheath; (c) EDS spectrum of region 1(core); (d) EDS spectrum of region 2 (sheath). 


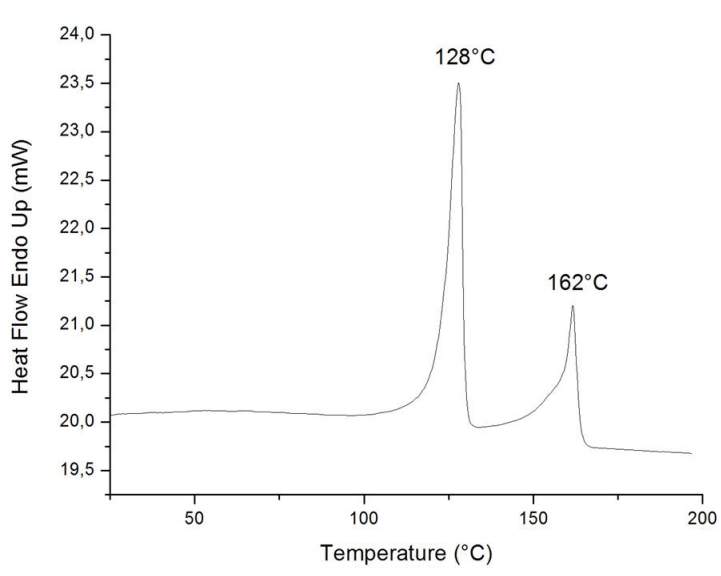

Figure 2. DSC thermogram of 3 denier PE/PP bicomponent fiber (nitrogen, second heating).

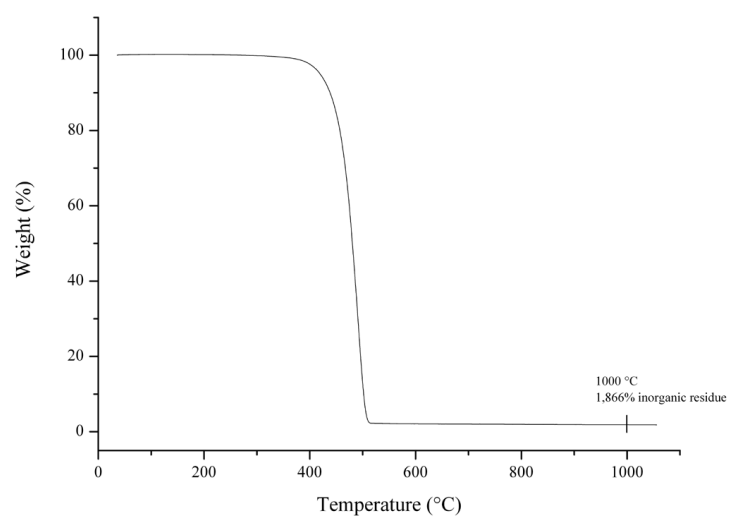

Figure 3. TGA curve of 3 denier PE/PP bicomponent fiber (nitrogen, $20^{\circ} \mathrm{C} / \mathrm{min}$, until $800^{\circ} \mathrm{C}$; synthetic air, $20^{\circ} \mathrm{C} / \mathrm{min}, 800^{\circ} \mathrm{C}$ $-1000^{\circ} \mathrm{C}$ and 2 minutes at $1000^{\circ} \mathrm{C}$ ).

$98.067 \%, 300^{\circ} \mathrm{C}-1000{ }^{\circ} \mathrm{C}$ and inorganic residue: $1.866 \%$, at $1000{ }^{\circ} \mathrm{C}$ (Figure 3). The low concentration of volatiles and presence of additives used to facilitate the processing of material and modify the physical chemical properties of final product, normally, consist in organic solvents that degrade and volatilize before PE and PP degradation. These polymers initiate the thermal degradation at the same range of temperature when submitted to TGA analysis, so it is not possible to verify using this technique the composition in $\%$ of each polymer contained in the fiber. On the other hand, it is possible to verify that there is an inorganic residue present in the fiber composition, which means that, can be the $\mathrm{TiO}_{2}$, a solid reagent very useful as filler in polymeric blends with properties to reduce transparency and turn the material more opaque as mentioned previously. In order to evaluate if this residue is $\mathrm{TiO}_{2}$, a typical analysis by pyrolysis with potassium pyrosulfate and posterior reaction with $\mathrm{H}_{2} \mathrm{O}_{2}$ was carried out.

\subsection{Characterization and quantification by colorimetric reaction}

The colorimetric technique allows evaluating the reaction of ion $\mathrm{Ti}^{+4}$ with $\mathrm{H}_{2} \mathrm{O}_{2}$ in sulfuric acid solution and evaluated by a calibration curve which obeys Lambert-Beer law (Lopes-Mollinero et al. $)^{[14]}$. The result achieved by this colorimetric reaction and the intensity of color read in UV-VIS spectrophotometer was $1.81 \%$ of $\mathrm{TiO}_{2}$. This data corroborated the value recorded in TGA analysis and confirmed presence of $\mathrm{Ti}^{+4}$.

\subsection{FT-IR characterization}

Currently, there are available many modes to obtain FT-IR spectrum and some of them were chosen to investigate if a particular mode can be correlated with the measures obtained by SEM. In this manner, the characteristics of surface analysis by DRIFT, UATR and PAS were discussed especially addressing the sheath layer to enhance the understanding some results found using other analytical techniques. The FT-IR transmission mode was also aim of this present study by pellet and casting film and using microscope.

Transmission mode: the transmission mode used in FT-IR with $\mathrm{KBr}$ pellet sample preparation consists in the simplest and more used in routine analysis of solid samples. Basically, the IR beam pass through the sample and the spectrum reveals the components of inner and surface sample, evidencing normally the material in major concentration. The use of $\mathrm{KBr}$ pellets has been avoided in a routine because demands time and the quality of preparation are very significant to achieve a good spectrum due the bands of humidity coming from hygroscopic property of $\mathrm{KBr}^{[15]}$. The 3 denier PE/PP bicomponent fiber was prepared with $\mathrm{KBr}(1: 400 \mathrm{mg})$ forming a pellet (Figure 4) and a thin film was also evaluated by transmission mode after dissolving the fiber in toluene lightly heated forming a casting film (Figure 5).

According to Figure 4, the transmission mode FT-IR spectrum of pellet defined mainly IR absorption bands related to PE and oxide: in $1470 \mathrm{~cm}^{-1}$ and $718 \mathrm{~cm}^{-1}$ (PE) and $500 \mathrm{~cm}^{-1}$ (oxide), marked with black stars. On the other hand, the spectrum of casting film by transmission (Figure 5) was crucial to define the presence of PP (marked with black stars, bands $1376,1167,997,973 \mathrm{~cm}^{-1}$ ) in the fiber since this polymer was not evidenced by pellet. In this case, the PE was also better defined and marked with red stars. These two sample preparations were chosen to attend the first classic mode to evaluate any material by FT-IR and compare this mode with other modern modes that provided more information about the composition of core and sheath, once the transmission mode is not selective to surface and analyze the sample as a whole. The importance to introduce the pellet was identified the oxide presence and the casting film allows to define the polymeric composition in the fiber.

In the Figure 4, the absorption bands in 3434 and $1631 \mathrm{~cm}^{-1}$ are due the moisture present in the pellet. However, it was noticed that this sample is very difficult to grind and, for consequence, the pellet formed is not homogenous due the difficult incorporation of it in the salt. This analysis suggested the presence of only one polymer in the fiber, 
PE. Since this technique has the drawback of moisture presence and difficulty to prepare a good pellet to analyze, other studies with different FT-IR techniques and accessories were carried out to drive this study to FT-IR analysis more reliable and feasible characterizing the composition of this bicomponent fiber.

Meanwhile, before starting the use of reflection and PAS techniques to analyze the polymers, the transmission mode using $\mathrm{KBr}$ pellet was also performed to verify the composition of the calcined residue of bicomponent fiber (Figure 6). It is possible to observe the presence of large bands at 671,500 and $422 \mathrm{~cm}^{-1}$, marked with black stars, that constitute characteristic bands of metallic oxide, as reference spectrum of $\mathrm{TiO}_{2}$, according to Hummel ${ }^{[16]}$. The spectrum shown in Figure 6 was compared to the Hummel ${ }^{[16]}$ reference spectrum that mentions two types of $\mathrm{TiO}_{2}$ crystals: rutile and anatase, spectrum number 5438 and 5439, respectively. Although the spectrum obtained from sample calcination presents the characteristic bands of anatase and rutile crystal formation, the bands present more similarity to anatase crystal. According to Chen et al. ${ }^{[17]}, \mathrm{TiO}_{2}$ consists in a multicrystal substance and the crystalline structures include brookite, anatase and rutile. Anatase and rutile are the structures more common to be used and they form crystalline structures in a tetragonal format and the rutile density is higher than anatase.

Valentim, Tavares \& Silva ${ }^{[18]}$ studied the effect of $\mathrm{TiO}_{2}$ addition in ethylene and vinyl acetate copolymer and characterized this mineral by transmission under $\mathrm{KBr}$ pellets. The result obtained in their study showed IR absorption bands in the same region of bands showed in Figure 6, as expected: about $669-555 \mathrm{~cm}^{-1}$ (regarding to Ti-O-Ti), and a band at $3450 \mathrm{~cm}^{-1}$, which the authors attributed to hydroxyl group present in the oxide surface or moisture of pellet. In this current study, this last band was also observed and the authors preferred to attribute it just to the presence of moisture, since there was observed a band at $1640 \mathrm{~cm}^{-1}$ which is also attributed to residue of water in the pellet. According to literature ${ }^{[15]}$ the transmission mode is not applicable to surface analysis of oxide.

FT-IR MIC transmission mode: this analytical technique uses a combination of FT-IR spectrometer with a microscope and is considered an important forensic tool that detects and identifies the fibers. FLYNN et al. evaluated the application of infrared chemical imaging to analysis of bicomponent fibers and they discussed about transmission and ATR mode using FT-IR MIC, given that ATR mode is more dedicated to surface analysis and transmission mode elucidates the sheath and core components by the spectrum combination of the both parts of bicomponent fibers ${ }^{[1]}$. This present study investigated the sample by FT-IR MIC transmission mode in order to compare the results obtained using FT-IR MIC to casting film spectrum. Indeed, the result obtained by FT-IR MIC transmission mode as shown in Figure 7 corroborates the information reported by FLYNN et al. about this specific mode, because the sample spectrum yielded a combined spectrum of sheath and core components. Furthermore, it is possible to assure that this analysis showed sensitivity similar of spectrum obtained by formation of casting film, once the bands related to PE and PP were very well defined

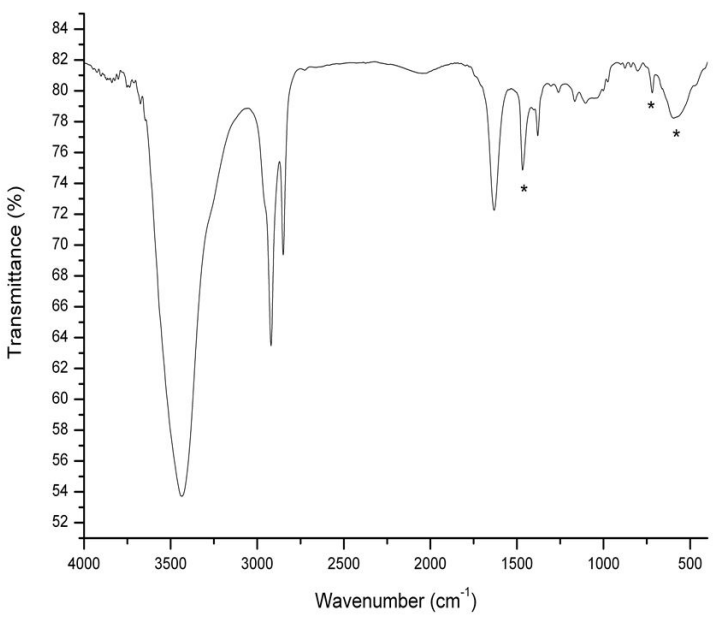

Figure 4. Transmission FT-IR spectrum of 3 denier PE/PP bicomponent fiber, as received, $\mathrm{KBr}$ pellet $(1: 400 \mathrm{mg})$. *Bands related to PE $\left(1470 \mathrm{~cm}^{-1}\right.$ and $\left.718 \mathrm{~cm}^{-1}\right)$ and to oxide $\left(500 \mathrm{~cm}^{-1}\right)$.

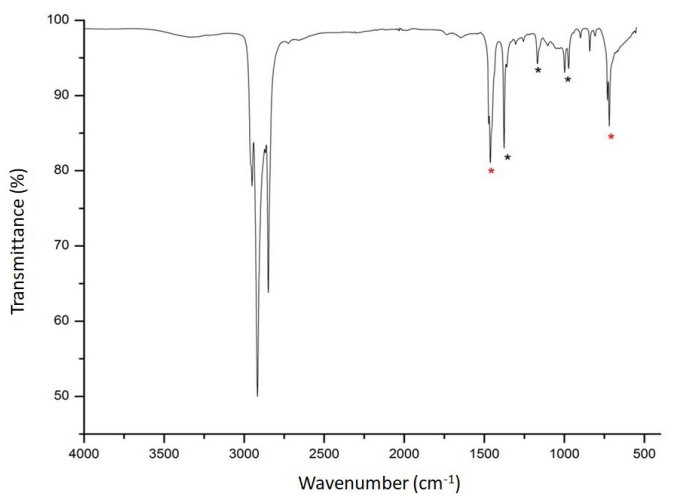

Figure 5. Transmission FT-IR spectrum of 3 denier PE/PP bicomponent fiber, after toluene treatment, casting film. Bands related to PE (red star) and Bands related to PP (black star).

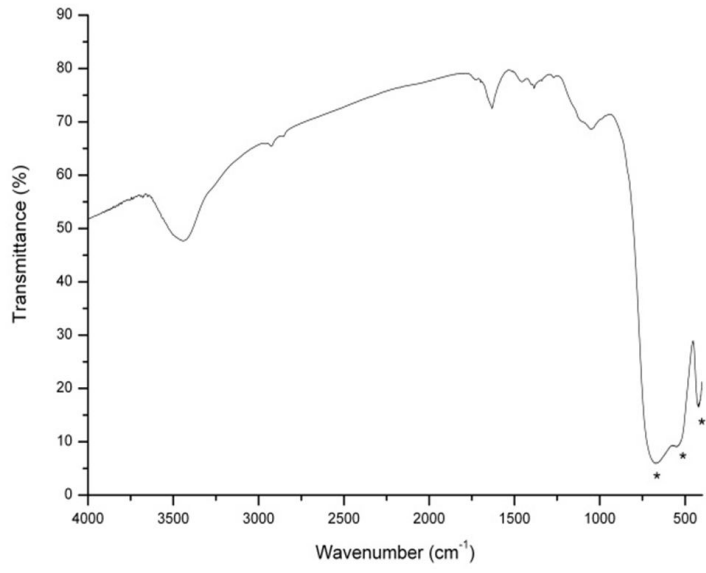

Figure 6. Transmission FT-IR spectrum of calcined residue of bicomponent fiber 3 denier PE/PP, KBr pellet (1:400mg). *Bands related to $\mathrm{TiO}_{2}$. 
and interference fringing, normally expected to transmission analysis using microscope, was not observed.

For this reason, the spectrum recognized PE (marked with red stars) and PP (marked with black stars) as the IR beam passed through the sheath and core of sample, as shown in Figure 7.

Reflection mode by DRIFT: Differently of transmission mode that use $\mathrm{KBr}$ pellet, the reflection mode by DRIFT do not require sample preparation, therefore the sample is analyzed directly by this technique without any treatment. Moreover, the main benefit of this technique is based on the intensification of non-fundamental bands (combination bands and overtones). So, a spectrum more completed of sample can be acquired by DRIFT mode, when compared to transmission mode providing more information about the sample ${ }^{[19]}$. However, as the interpretation of overtones and combination bands is not easy, the presence of these bands can cause a disturbance the process of spectrum interpretation. Generally, DRIFT spectra evidence surface coating and inner composition showing, in some spectra, large bands. In this case, the spectrum presented well-defined bands suggesting that the IR beam passed through by the surface in a depth with more $3 \mu \mathrm{m}$, according to results obtained by SEM images. The DRIFT spectrum shown in Figure 8, evidenced with excellent resolution, the presence of PE (duplet about 720 and $730 \mathrm{~cm}^{-1}$, marked with red star) and PP $\left(1167,997,973,841 \mathrm{~cm}^{-1}\right.$, marked with black stars). As from this data, it is possible to confirm the presence of PP in the sample as supplier information, but it is unknown what polymer consists in the sheath and core by this spectrum. Furthermore, the spectrum also evidenced the presence of $\mathrm{TiO}_{2}$ once there was observed a large absorption band at $555 \mathrm{~cm}^{-1}$. The DRIFT analysis allowed to check the presence of $\mathrm{PE}, \mathrm{PP}$ and $\mathrm{TiO}_{2}$ and consists in a good technique to analyze globally the fibers. For this reason, DRIFT definitely is not considered a selective technique.

UATR reflection mode: the UATR reflection mode was used by Noureddine et al. ${ }^{[20]}$ in studies of chemical coating in woven samples. This is considered a surface and non-destructive technique that use a zinc selenide crystal with diamond to analyze the surface material pressuring the sample against the crystal to assure that it has a good contact with the surface of sample and the incident IR beam, preventing loss of beam irradiation. As DRIFT technique, UATR does not require sample preparation and the sample can be analyzed directly. With the purpose to investigate the surface or the sheath of the bicomponent fibers, the result found of sample presented a typical spectrum of PE being observed characteristic bands of PE: $1470 \mathrm{~cm}^{-1} \mathrm{e}^{2} 29 \mathrm{~cm}^{-1}$, marked with red stars. This result allows concluding that this polymer composes the sheath of fiber (Figure 9), once this technique examines the superficial part of the sample. According to data sheet of FT-IR equipment supplier, the zinc selenide and diamond crystal has $2.0 \mu \mathrm{m}$ of IR beam penetration depth. Once the image (b) of Figure 1 (SEM) showed the width of external layer measures about $3.7 \mu \mathrm{m}$, it is clear that the IR beam penetrates the external side of fiber and did not reach the core of fiber, for this reason, the measures obtained by SEM were important to comprehend this surface mode and the spectrum resulted by UATR has corroborated the information described by equipment supplier.

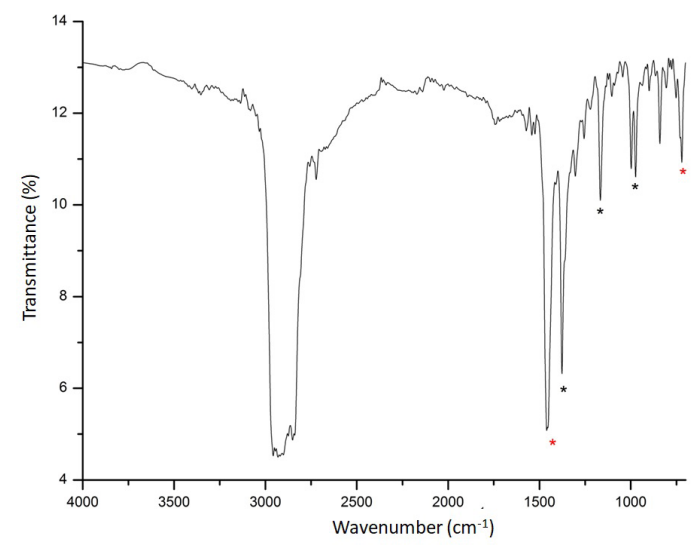

Figure 7. FT-IR MIC transmission mode spectrum. Bands related to PE (red star) and bands related to PP (black star).

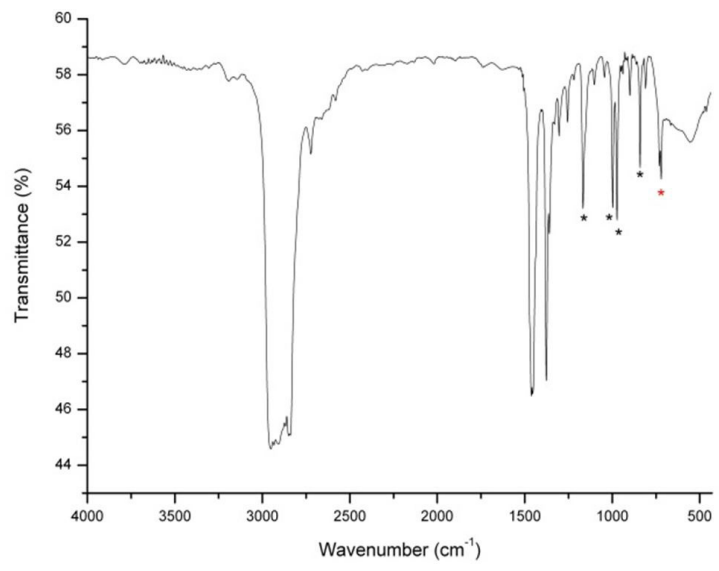

Figure 8. DRIFT spectrum of 3 denier PE/PP bicomponent fiber, as received. Bands related to PE (red star) and bands related to PP (black star).

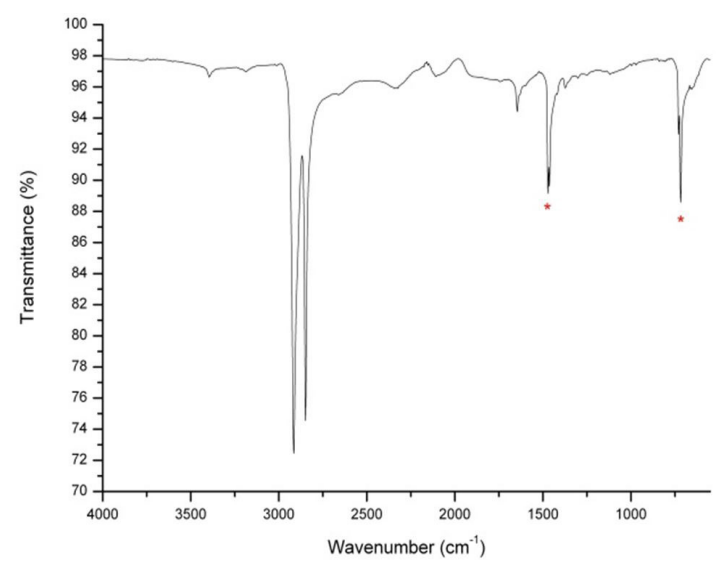

Figure 9. UATR spectrum of 3 denier PE/PP bicomponent fiber, as received. Bands related to $\mathrm{PE}$ (red star). 
In other words, UATR technique showed more selectivity to evaluate the $\mathrm{PE}$ in bicomponent fiber and confirm the presence of this polymer in the sheath.

PAS mode: the PAS mode is recognized as a near-surface and non-destructive analytical technique. This technique is based on the direct measure of absorbed energy by samples through a photoacoustic signal less susceptible to light scattering of particles present in the material ${ }^{[21,22]}$. The IR beam is absorbed by sample creating an induced heating due the absorption which provide a thermal expansion modifying the pressurization of inert gas present within the chamber and transforming this alteration of pressure in a photoacoustic signal detected by one microphone and then a spectrum is generated by a computer system. PAS represents a good technique to perform dark samples once as higher the rate of IR absorption better will be the photoacoustic signal ${ }^{[23]}$. Faster mirror velocities yield lower-intensity signals because the thermal diffuse depth decreases ${ }^{[24]}$. For this reason, more superficial layer has better resolution with increase of mirror velocity. The spectrum obtained of bicomponent fibers using PAS technique also include $100 \% \mathrm{PE}$ and $100 \%$ PP materials used as standard (Figure 10). The image suggests that at slow velocity $(0.05 \mathrm{~cm} / \mathrm{s})$ it is possible to observe the presence of PP, characteristics bands marked with black stars, corroborating the result found by DRIFT mode. Moreover, when the $1.00 \mathrm{~cm} / \mathrm{s}$ is applied, the superficial layer is more evidenced and the spectra obtained are typical of PE, characteristics bands marked with red stars, as reported in UATR mode.

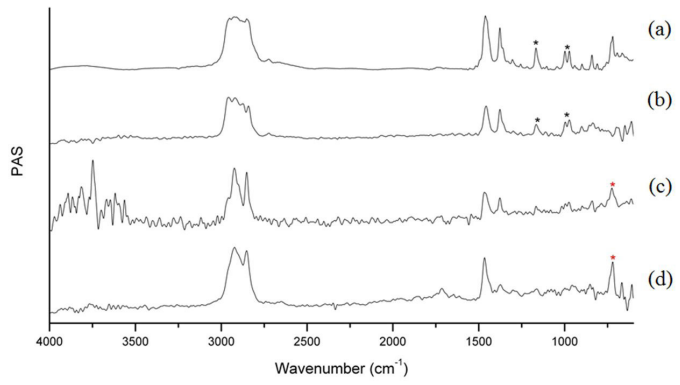

Figure 10. PAS spectrum: (a) 3 denier PE/PP bicomponent fiber, as received, velocity $=0.05 \mathrm{~cm} / \mathrm{s}$; (b) $100 \%$ PP material - Velocity $=0.05 \mathrm{~cm} / \mathrm{s}$; (c) 3 denier PE/PP bicomponent fiber, as received, velocity $=1.00 \mathrm{~cm} / \mathrm{s}$; (d) $100 \% \mathrm{PE}$ material velocity $=0.05 \mathrm{~cm} / \mathrm{s}$. Bands related to PE (red star) and bands related to PP (black star).

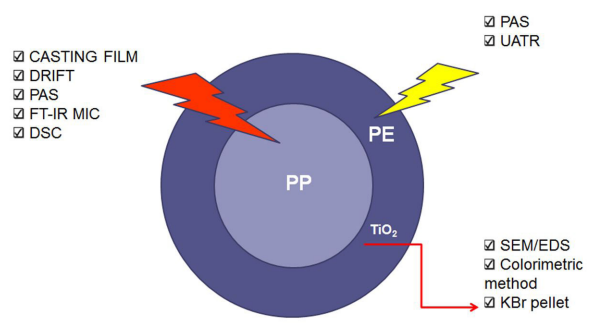

Figure 11. Representative figure of 3 denier PE/PP bicomponent fiber showing the cross section of fiber and analytical techniques used to characterize $\mathrm{PE}, \mathrm{PP}$ and $\mathrm{TiO}_{2}$ in each bicomponent fiber section.
Based on these results, a comprehensive study for characterization of bicomponent fiber can be summarized by a representative figure (Figure 11) which compiles the analytical methodologies used to determine the respective polymer and oxide that composed each part of fiber (sheath and core).

\section{Conclusions}

This present study started the elucidative process of 3 denier PE/PP bicomponent fiber with an analysis of imaging able to obtain a spatial configuration of fiber that clearly defined the fiber as the sheath-core structure. The EDS analysis was fundamental to prove that $\mathrm{TiO}_{2}$ was added in PE bulk to compose the sheath. A non-destructive technique, the FT-IR analyses were essential to characterize each part of sample using modern accessories and, from the results obtained in this study, FT-IR spectroscopy can be considered the most important analytical technique to elucidate the polymer composition of each section of sheath-core bicomponent fibers. Once this study was focused on using different modes of FT-IR, it was possible to conclude that the feasible techniques to analyze the polymers of sheath (PE) and core (PP) structures were UATR and PAS. UATR was able to identify the component of more external layer and PAS revealed the components of both sheath and core, using different velocities. Other modes as transmission and DRIFT confirmed the presence of PE and PP in the whole fiber. Overall, this study provided a sequence of analytical methodologies that can be very helpful to understand since the spatial configuration until the real composition of sheath-core bicomponent fibers.

This study disclosed about the important role of FT-IR spectroscopy to elucidate the composition of sheath-core structure along with other analytical methods such as: SEM/EDS and DSC. Colorimetric assay was also conducted to confirm the presence of titanium detected by the EDS spectrum and the concentration of this inorganic residue found by TGA.

\section{Acknowledgements}

This study was supported in part by the National Senior Visiting Professor Program (PVNS) from the Coordenação de Aperfeiçoamento Pessoal de Nível Superior (CAPES). The authors also would like to thank Fabio Eduardo Rangel for the support and collaboration in this study.

\section{References}

1. Flynn, K., O’Leary, R., Roux, C., \& Reedy, B. J. (2006). Forensic analysis of bicomponent fibers using infrared chemical imaging. Journal of Forensic Sciences, 51(3), 586-596. http:// dx.doi.org/10.1111/j.1556-4029.2006.00116.x. PMid:16696706.

2. Dasdemir, M., Maze, B., Anantharamaiah, N., \& Pourdeyhimi, B. (2012). Influence of polymer type, composition, and interface on the structural and mechanical properties of core/sheath type bicomponent nonwoven fibers. Journal of Materials Science, 47(16), 5955-5969. http://dx.doi.org/10.1007/s10853-0126499-7.

3. Andrzejewski, J., Szostak, M., Krasucki, J., Barczewski, M., \& Sterzyński, T. (2015). Development and characterization of the injection-molded polymer composites made from bicomponent 
fibers. Polymer-Plastics Technology and Engineering, 54(1), 33-46. http://dx.doi.org/10.1080/03602559.2014.935414.

4. Demirci, E., Acar, M., Pourdeyhimi, B., \& Silberschmidt, V. V. (2011). Finite element modelling of thermally bonded bicomponent fibre nonwovens: Tensile behavior. Computational Materials Science, 50(4), 1286-1291. http://dx.doi.org/10.1016/j. commatsci.2010.02.039.

5. McIntyre, J. E. (2004). Synthetic fibres: nylon, polyester, acrylic, polyolefin. Cambridge: Woodhead Publishing Ltd. http://dx.doi.org/10.1201/9780203501702.

6. Wang, Y., Wang, L., He, X., Zhang, Z., Yu, H., \& Gu, J. (2014). Integration of RAFT polymerization and click chemistry to fabricate PAMPS modified macroporous polypropylene membrane for protein fouling mitigation. Journal of Colloid and Interface Science, 435, 43-50. http://dx.doi.org/10.1016/j. jcis.2014.08.013. PMid:25217729.

7. Wu, R. Y., Chu, C. W., Chen, S. H., \& Chiang, C. Y. (2010). US Patent 7781059 B2. Taiwan: Far Eastern Textile Ltd. Retrieved in 2016, February 09, from https://www.google. com.ar/patents/US7781059.

8. Meleiro, P. P., \& García-Ruiz, C. (2015). Spectroscopic techniques for the forensic analysis of textile fibers. Applied Spectroscopy Reviews, 51(4), 258-281.

9. Sionkowska, A. (2011). Current research on the blends of natural and synthetic polymers as new biomaterials: review. Progress in Polymer Science, 36(9), 1254-1276. http://dx.doi. org/10.1016/j.progpolymsci.2011.05.003.

10. Prati, S., Rosi, F., Sciutto, G., Mazzeo, R., Magrini, D., Sotiropoulou, S., \& Van Bos, M. (2012). Evaluation of the effect of six different paint cross section preparation methods on the performances of Fourier Transformed Infrared microscopy in attenuated total reflection mode. Microchemical Journal, 103, 79-89. http://dx.doi.org/10.1016/j.microc.2012.01.007.

11. Burdet, P., Croxall, S. A., \& Midgley, P. A. (2015). Enhanced quantification for 3D SEM-EDS: using the full set of available X-ray lines. Ultramicroscopy, 148, 158-167. http://dx.doi. org/10.1016/j.ultramic.2014.10.010. PMid:25461593.

12. Jiang, Z., Tijing, L. D., Amarjargal, A., Park, C. H., An, K. J., Shon, H. K., \& Kim, C. S. (2015). Removal of oil from water using magnetic bicomponent composite nanofibers fabricated by electrospinning. Composites. Part B, Engineering, 77, 311318. http://dx.doi.org/10.1016/j.compositesb.2015.03.067.

13. Turi, E. A. (1997). Thermal characterization of polymer materials. New York: Academic Press Inc.

14. Lopez-Molinero, A., Liñan, D., Sipiera, D., \& Falcon, R. (2010). Chemometric interpretation of digital image colorimetry. application for titanium determination in plastics. Microchemical Journal, 96(2), 380-385. http://dx.doi.org/10.1016/j.microc.2010.06.013.
15. Silverstein, R. M., Webster, F. X., \& Kiemle, D. J. (2005). Spectrometric identification of organic compounds. New York: Wiley.

16. Hummel, D. O., \& School, F. (1984). Atlas of polymer and plastics analysis (Vol. 3). Weinheim: Verlag Chemie.

17. Chen, X. D., Wang, Z., Liao, Z. F., Mai, Y. K., \& Zhang, M. Q. (2007). Roles of anatase and rutile $\mathrm{TiO}_{2}$ nanoparticles in photooxidation of polyurethane. Polymer Testing, 26(2), 202208. http://dx.doi.org/10.1016/j.polymertesting.2006.10.002.

18. Valentim, A. C. S., Tavares, M. I. B., \& Silva, E. O. (2014). Efeito da adição de $\mathrm{TiO}_{2}$ nas propriedades térmicas e na cristalinidade do copolímero de etileno/acetate de vinila. Quimica Nova, 37(2), 255-259.

19. Arrizabalaga, I., Gomez-Laserna, O., Aramendia, J., Arana, G., \& Madariaga, J. M. (2014). Determination of the pigments present in a wallpaper of the middle nineteenth century: The combination of mid-diffuse reflectance and far infrared spectroscopies. Spectrochimica acta. Part A, Molecular and Biomolecular Spectroscopy, 124, 308-314. http://dx.doi. org/10.1016/j.saa.2014.01.017. PMid:24503152.

20. Abidi, N., Hequet, E., Turner, C., \& Sari-Sarraf, H. (2005). FTIR analysis of crosslinked cotton fabric using a $\mathrm{ZnSe}$-universal attenuated total reflectance. Journal of Applied Polymer Science, 96(2), 392-399. http://dx.doi.org/10.1002/app.21449.

21. Lu, Y., Du, C., Yu, C., \& Zhou, J. (2014). Classifying rapeseed varieties using Fourier transform infrared photoacoustic spectroscopy (FTIR-PAS). Computers and Electronics in Agriculture, 107, 58-63. http://dx.doi.org/10.1016/j.compag.2014.06.005.

22. Yang, C. Q. (1992). Infrared Spectroscopic Analysis of Textile Materials Degradation Using Photoacoustic Detection. Industrial \& Engineering Chemistry Research, 31(2), 617-621. http:// dx.doi.org/10.1021/ie00002a026.

23. Peltre, C., Bruun, S., Du, C., Thomsen, I. K., \& Jensen, L. S. (2014). Assessing soil constituents and labile soil organic carbon by midinfrared photoacoustic spectroscopy. Soil Biology \& Biochemistry, 77, 41-50. http://dx.doi.org/10.1016/j. soilbio.2014.06.022.

24. Bhardwaj, N. K., \& Nguyen, K. L. (2007). Photoacoustic Fourier transform infrared spectroscopic study of hydrogen peroxide bleached de-inked pulps. Colloids and Surfaces. A, Physicochemical and Engineering Aspects, 301(1-3), 323-328. http://dx.doi.org/10.1016/j.colsurfa.2006.12.077.

Received: Mar. 08, 2016

Revised: July 06, 2016

Accepted: Sept. 08, 2016 